\title{
Estrategias de flexibilidad productiva y cambio tecnológico en Teléfonos de México
}

\author{
Gabriel PéReZ* y Gerardo TunAL **
}

\begin{abstract}
This paper analyses how the process of productive restructuring and labour flexibility took place in Teléfonos de México S.A. de C.V. (Telmex) starting in the decade of 1980. Specifically, it examines how the process was given through an agreement to allow opening and competition, it was also possible thanks to the changes in some clauses of the Collective Work Contracts (ССТ) derived from the application of an agreement of productivity and specialisation as well as development strategies and the role that Sindicato de Telefonistas de la República Mexicana (STRM) played. We also study the impact of the policies of labour flexibility in the hiring processes of Telmex. At the same time, we analyse the reach of the modernisation process to understand this restructuring.
\end{abstract}

Keywords: productive restructuring, labor flexibility, telephone services, work organisation.

\section{Resumen}

El presente artículo analiza cómo se dio el proceso de reestructuración productiva y flexibilidad laboral en Teléfonos de México, s.A. de C.v. (Telmex) a partir de los años ochenta. Específicamente se examina cómo se da éste a través de la firma de un convenio de concertación para la apertura y la competencia; los cambios en algunas cláusulas de los Contratos Colectivos de Trabajo (ССТ) derivadas de la puesta en marchar de un convenio de productividad; la compactación de especialidades; las estrategias de desarrollo, y del papel que jugó el Sindicato de Telefonistas de la República Mexicana (STRM). Asimismo, se estudia el impacto que tuvo la puesta en marcha de políticas de flexibilización laboral en la contratación colectiva de Telmex y los alcances del proceso de modernización, con la finalidad de hacer algunas reflexiones generales sobre la reestructuración productiva emprendida por esta empresa.

Palabras clave: reestructuración productiva, flexibilidad laboral, servicios telefónicos, organización del trabajo.

* Universidad Iberoamericana y Universidad Nacional Autónoma de México, correo-e: gabriel_perez@hotmail.com

** Instituto Tecnológico de Estudios Superiores de Monterrey y Universidad Nacional Autónoma de México, correo-e: gertunsa@yahoo.com.mx 


\section{Introducción}

Es un hecho que a partir de los primeros años de la década de los ochenta del siglo xx los principales países capitalistas de occidente comenzaron a experimentar, con distintos matices y ritmos, un proceso de descomposición en sus modelos de acumulación, que los orilló a replantear nuevas formas de producción en la dinámica de la globalización, la competencia y la apertura de los mercados. De igual forma, este tránsito a nuevos paradigmas productivos implicó nuevas formas de organización del trabajo, de tal suerte que los objetivos inherentes a esta nueva lógica, tuvieron su efecto más inmediato en las organizaciones productivas. Lo anterior provocó reacciones encontradas entre las gerencias, los líderes sindicales, el Estado y, en mayor medida, entre los trabajadores.

Los retos planteados por la modernización productiva, sugerían la vuelta y el replanteamiento de la filosofía gerencial japonesa de los años cincuenta del siglo xx. Es así como en muchas de las empresas del mundo se empezó a poner en marcha una nueva cultura laboral propia de los nuevos modelos de organización del trabajo. Resulta evidente que no todas las empresas ni todos los sectores económicos estaban preparados para el proceso de modernización productiva que exigía la racionalidad universal del mundo globalizado, de tal suerte que sólo las empresas y los sectores que habían demostrado en el pasado un impacto positivo en el Producto Interno Bruto (PIB) y se habían posicionado como sectores económicamente prioritarios, estaban en el nuevo juego de la economía neoliberal.

Particularmente, el sector telefónico en México había presentado una trayectoria de mediana competitividad y flexibilidad a los cambios estructurales que había experimentado la economía mundial hasta antes de la crisis del modo de acumulación fordista. La particularidad de este sector es que estaba concentrado en una sola empresa, la cual había monopolizado la producción y la venta de los servicios telefónicos. El caso de Teléfonos de México (en lo sucesivo Telmex) sin duda alguna, representa un caso muy especial en la economía y los sistemas de relaciones industriales en México, en tanto que dicha empresa se había ganado el visto bueno del Estado y representaba una empresa muy atractiva para los inversionistas nacionales y extranjeros.

La importancia del análisis de la reestructuración productiva vía la flexibilidad laboral en Telmex radica en darle seguimiento a dicho proceso, para verificar y constatar que no se trata sólo de 
un caso exitoso en una coyuntura particular, sino de un evento que si bien se ha ido adaptando a nuevas y diferentes coyunturas, ha evidenciado lo que no se ha dado como norma en la mayoría de las empresas mexicanas, es decir, el debilitamiento del problema de la posible relación entre el cambio en la estructura de las ocupaciones -con la heterogeneidad de intereses de los empleados, que conduciría hacia la fragmentación en las formas de organización y a la descentralización de sus negociaciones con la gerencia (De la Garza, 2002: 10)-y la articulación entre estructuras, identidades y acciones colectivas.

Pese a que, desde los primeros años de la década de los ochenta del siglo pasado y hasta la fecha, se han estudiado los procesos de modernización y flexibilidad laboral derivados del cambio en el modo de acumulación, creemos que es pertinente seguir analizando dichos procesos, específicamente el caso Telmex, en tanto que actualmente en muchos países de América Latina se está presenciando una adecuación a nuevos esquemas regulatorios derivados de la sustitución de estructuras monopolizadas que responde a situaciones de apertura comercial. De igual forma, es evidente que en el mundo actual la rama de las telecomunicaciones representa un sector de mucha importancia para cualquier nación, en tanto que éste contribuye de manera directa al desarrollo económico y social de dichas naciones.

El objetivo principal de este trabajo estará orientado a hacer una revisión y una reflexión de cómo Telmex inició un proceso de reestructuración productiva, vía la flexibilidad laboral. En una primera parte se analizará cómo se dio el proceso de modernización en Telmex, específicamente se hará énfasis en la manera en que la firma de un Convenio de Concertación para la Modernización en Telmex dio paso a la apertura a la competencia en dicha empresa. De igual forma, se examinarán los principales cambios en el Contrato Colectivo de Trabajo (ССт) y lo que significó la firma de un Convenio de Productividad en Telmex. En una segunda parte se investiga, de manera más puntual, cómo se da la flexibilidad laboral en el сст de Telmex a partir de 1994 y hasta el año 2000, y se hace énfasis en aquellos aspectos que podrían dar evidencia de que en esta empresa se desarrollaron políticas orientadas a la aplicación de formas flexibles de producción. Finalmente, y a manera de corolario, se hace una reflexión sobre lo que implicó en Telmex el proceso de reestructuración productiva vía la flexibilidad laboral. 


\section{El proceso de modernización de Telmex}

En los últimos años el sector de las telecomunicaciones ha incrementado de manera sostenida su importancia en el conjunto de la planta productiva del país. Las telecomunicaciones se han expandido en forma permanente y sus unidades productivas no sólo tienden a ser más grandes sino que atraviesan por un proceso de modernización tecnológica-organizativa y de privatización que adelgaza paulatinamente el grueso de las empresas paraestatales. Sin duda alguna este es el caso de Telmex.

Telmex, junto con sus empresas subsidiarias y asociadas, proporciona servicios de telecomunicaciones en los ámbitos nacional e internacional a clientes residenciales y comerciales que operan en una amplia gama de actividades. Actualmente es el proveedor más importante de servicios de telefonía local y de larga distancia en todo México. A principios de los años ochenta del siglo xx se había convertido, aparentemente, en una industria obsoleta y sumamente retrasada en cuanto a la aplicación de tecnología digital en los procesos de trabajo que ya se manejaban en el ámbito mundial. Por tal razón adoptó, desde la segunda mitad de los años ochenta del siglo pasado, una estrategia para enfrentar sus problemas tanto productivos como laborales. Esta estrategia consistió en la expansión, fragmentación y especialización de algunos de los procesos de trabajo mediante el uso creciente de empresas filiales o subsidiarias y de terceras compañías. Es precisamente en este tipo de empresas en las que Telmex se ha apoyado para ofrecer nuevos servicios, así como para crecer como empresa y modernizarse.

El actual proceso de modernización de Telmex ocurrió básicamente a partir de su privatización a finales de los años ochenta del siglo pasado. Atrás de esta medida estaban dos hechos de consideración: primero, las grandes deficiencias de la comunicación nacional que provocaban un reclamo unánime, no sólo de las empresas sino de la población en general, por contar con un servicio más eficiente; segundo, la necesidad de capital urgente para llevar a cabo la modernización digital, dado que el Estado se mostraba carente de recursos para una inversión mínima de 10 mil millones de dólares.

La lógica de la privatización en este caso apunta más a la tendencia, ya manifiesta en los países industrializados, de desregulación de la economía en un ámbito que ya se sitúa como estratégi- 
co para la producción mundial. En este sentido, el modelo económico mexicano de apertura comercial basado en la atracción de capital extranjero exigía mayor eficiencia en las comunicaciones, no tanto en beneficio del público consumidor ordinario, sino de los llamados grandes usuarios que demandaban servicios más avanzados, rápidos, mejores y más diversificados. Es importante recordar que, en México, los primeros demandantes fueron los sistemas de banca y crédito, la industria maquiladora y, en general, las grandes industrias exportadoras (Medina, 1995: 141).

A partir de la preocupación por modernizar y aumentar la productividad de Telmex, durante 1989 se produjeron cambios importantes en su organización administrativa. Como parte de éstos, el 14 de abril del mismo año se firmó un convenio de concertación entre empresa, sindicato y el gobierno federal que establecía el compromiso de la empresa de discutir con el Sindicato de Telefonistas de la República Mexicana (STRM) un documento en el que se proponía la modificación de algunas cláusulas del ССт, la sustitución de los convenios departamentales por los perfiles de puesto, y la transformación de la estructura organizativa. Según la empresa, esta disposición obedeció, esencialmente, a la necesidad de modernizarse y de prestar un servicio eficiente acorde con la evolución tecnológica, que en forma constante se venía presentando en el ramo de las telecomunicaciones y con motivo de múltiples quejas que los usuarios habían presentado tanto a la Secretaría de Comunicaciones y Transportes (SCT), como a la Procuraduría Federal del Consumidor (PROFECO) sobre deficiencias en el servicio.

El 18 de septiembre de 1989, el ex presidente Carlos Salinas de Gortari, en una asamblea del sTRM, anunció la oferta de dicha empresa a la iniciativa privada, argumentando que se trataba de una decisión coherente con la nueva política económica. En ese mismo año el Programa de Modernización de Telecomunicaciones, dado a conocer por la SCT, aclaró que la administración de la red y su expansión quedaría bajo el dominio público y, por lo tanto, no se admitiría la existencia de redes de competencia. Fuera de la red de telefonía para servicio local o de larga distancia, todos los demás servicios se ofrecerían en abierta competencia bajo un régimen de concesiones (Sandoval, 1990: 53).

Con este programa, el Estado permanecería sólo en los espacios de la regulación de la competencia y dejaría en manos de esta última la reestructuración del sistema de telecomunicaciones. Las bases del programa se resumen así: garantizar que el Estado man- 
tenga la rectoría en las telecomunicaciones del país, modernizar y mejorar la calidad de los derechos de los trabajadores. Los inversionistas deberían respetar estos términos, según el Convenio de Concertación para la Modernización que se firmó (Sandoval, 1990: 54). Por su parte, Telmex ha reiterado en numerosas ocasiones que son una pieza clave para el desarrollo de la nación, en tanto que las telecomunicaciones constituyen el camino más corto para alcanzar el desarrollo y, por lo tanto, es bueno contar con una relación adecuada y positiva con el Estado (Vega, 1994: 8).

Desde el anuncio de la privatización de Telmex, ha puesto un especial énfasis en la extensión y modernización de su red telefónica. Se ha avanzado considerablemente en el tendido del circuito de larga distancia, en el número de aparatos telefónicos y de líneas atendidas. Telmex ha llevado a cabo una profunda transformación tecnológica al instalar nuevas centrales; redes telefónicas y planta exterior; larga distancia; repetidoras; materiales para instalaciones terminales; equipo para mejorar el servicio; un proyecto de la instalación de un cable submarino de fibra óptica entre México, Estados Unidos y Europa, y la instalación de 13,500 $\mathrm{km}$ de cable de fibra óptica en todo el país para agilizar el tráfico telefónico de larga distancia nacional e internacional. ${ }^{1}$

Para 1992, y sin interrumpir el servicio, Telmex inició la sustitución total de centrales análogas por centrales digitales, lo que provocó efectos significativos en la organización del trabajo en la empresa y en las relaciones en su interior. Los cambios ocurridos se inscriben dentro de un contexto de modernización relevante en el mundo del trabajo, que tiene dimensiones globales. En esto radica la importancia del proceso de modernización en Telmex durante el periodo que se analiza.

En este contexto de cambios, se incorporó una nueva situación contractual que favorecía el logro de una mayor productivi-

\footnotetext{
${ }^{1}$ La tecnología de fibra óptica es de las más avanzadas que existen en materia de telecomunicaciones en el ámbito internacional, en tanto que un solo par de fibras puede cursar 30 mil conversaciones telefónicas simultáneas. Las fibras ópticas logran disminuir, casi en su totalidad, ruidos e interferencias hasta multiplicar las formas de envío y recepción de información vía telefónica. Dichas fibras están constituidas por filamentos de vidrio de alta pureza extremadamente compactas y están fabricadas a base de silicio. Para la instalación de los $13,500 \mathrm{~km}$ de fibra óptica que sirvieron para modernizar el sistema y servicio de larga distancia nacional e internacional, Telmex suscribió convenios con las empresas AT\&T y Alcatel-Indetel. Según estos acuerdos, AT\&T proporcionó los cables de fibra óptica y sus componentes, mientras que su subsidiaria en España proporcionó el equipo de transmisión. La participación total de AT\&T en este contrato se estableció en $60 \%$ del total; en este sentido, la participación de Alcatel-Indetel fue de $40 \%$ en relación con el programa total para la instalación de la red de fibra óptica (Telmex, 1991: 6-7).
} 
dad. Tal tendencia de la empresa era respaldada por el sindicato y consistía en establecer como prioridad fundamental la productividad y calidad en el servicio y la modernización tecnológica de las telecomunicaciones; se concretó de forma más acabada dentro de los lineamientos marcados en el сCт 1992-1994, y de los cuales, además de las modificaciones señaladas anteriormente, destacan la de las cláusulas 9, 27, 193, 194 y 195 que impulsan, por un lado, una mayor productividad de los trabajadores y, por otro, la implantación de proyectos de modernización tecnológica por parte de la empresa. ${ }^{2}$ Estos cambios contractuales sirvieron de base para poner en marcha el Programa de Calidad y Productividad a finales de octubre de 1992, el cual se proponía acelerar la solución de los problemas del servicio telefónico en todo el país.

Es importante tener en cuenta que la mayoría de los trabajadores de Telmex fueron contratados durante los años setenta o prin-

${ }^{2}$ La cláusula 9 menciona que la empresa y el sindicato reconocen como interés fundamental de sus trabajos asegurar la prestación de un servicio telefónico de la mejor calidad y en la mayor amplitud al público usuario, al menor costo. Por ello se reconoce la necesidad imprescindible de incrementar la capacitación de personal, mejorar la eficiencia utilizando métodos modernos de administración y, en general, hacer uso de la más alta tecnología en beneficio del público. Asimismo, en tal cláusula se cambia la terminología con respecto a que empresa y sindicato aseguran la prestación de un servicio de telecomunicaciones, antes se decía que daban servicio telefónico, ahora se habla en plural y en un sentido más amplio, apegados a la expansión de nuevos servicios en comunicación; de igual forma, se cambió la referencia de un público usuario, a cliente, con una terminología más apegada a la filosofía cliente-proveedor, en términos empresariales. En la cláusula 27 se agrega un inciso bajo la letra 'g', que dice que en todos los casos la contratación de nuevo personal de confianza o sindicalizado deberá efectuarse, en las localidades, departamentos y en los tiempos en que se requiere, de acuerdo con la productividad y las necesidades del servicio. Por lo que respecta a la cláusula 193, se establecieron los siguientes lineamientos: cuando la empresa decida implantar los proyectos de modernización y/o nueva tecnología que juzgue convenientes, informará amplia y oportunamente sobre los mismos al sindicato para efectos de difusión y capacitación del personal sindicalizado que sea requerido, quien deberá realizar y atender todas las funciones y labores inherentes que se deriven de dichos proyectos. La capacitación se llevará a cabo conforme lo requiera la introducción de los nuevos proyectos o equipos. De los cambios mencionados destaca la imposición unilateral por parte de la empresa, para la implantación de nuevas tecnologías, y su compromiso de sólo avisar al sindicato de los cambios aceptados. Además de transformar los convenios departamentales en especialidades, con sus respectivos perfiles de puestos y categoría, también se formalizan mecanismos de movilidad y flexibilización en la organización del trabajo. Por su parte la cláusula 194 hace énfasis en que la empresa y el sindicato deben estar de acuerdo para alcanzar niveles internacionales que, en materia de capacitación y productividad, tienen las administraciones de empresas de telecomunicaciones más competitivas del mundo. A su vez, la cláusula 195 establece que la empresa y el sindicato acuerdan un programa para la permanente elevación y mejoramiento de la calidad y productividad en las diferentes áreas que la conforman, considerando que la productividad es consecuencia de la óptima articulación entre el incremento cuantitativo y cualitativo de la producción, el mejoramiento de la calidad de las condiciones de trabajo y de vida, así como de la capacitación productiva del propio trabajador. 
cipios de los ochenta del siglo xx, cuando la compañía era una empresa pública y monopolio de las telecomunicaciones en México, lo que permitía mayor estabilidad en el empleo. Estos mismos trabajadores siguen actualmente laborando aunque con algunos cambios de puesto o jubilaciones.

La empresa Telmex ha sostenido en diversas ocasiones que su desarrollo está basado en el compromiso con sus trabajadores porque, a diferencia de lo que pasa con el resto de las compañías de telecomunicaciones en el mundo, ha elegido la estrategia de conservar su planta laboral, estableciendo un sistema de recompensas, salarios y productividad bajo esquemas flexibles que permiten motivar y alentar al personal otorgándoles seguridad en el empleo. Este tipo de estrategia le ha servido a Telmex para no encontrar resistencia de los trabajadores para realizar los cambios necesarios para su modernización; sin embargo, es muy probable que en el entorno de apertura a la competencia lo impulse en un futuro no muy lejano a realizar recortes de personal, para permitir mayores niveles de flexibilidad y productividad.

Para el 26 de abril de 1993 y teniendo como testigos de honor al presidente de ese momento, Carlos Salinas de Gortari, al presidente del Consejo de Administración de Telmex, Carlos Slim Helú; y al Secretario General del STRM, Francisco Hernández Juárez, se ratificó el compromiso de establecer relaciones laborales productivas con un nuevo Convenio de Productividad que se enmarcaría dentro del Programa General Permanente de Incentivos a la Productividad de Telmex. Los objetivos del nuevo convenio estaban relacionados con: i) la satisfacción de las demandas del usuario y la oferta de mejores servicios telefónicos; ii) el cumplimiento de las metas establecidas tanto por el Título de Concesión como por la empresa; iii) el aumento de los niveles de producción de la empresa a través de incrementos de la productividad de la mano de obra; iv) propiciar el trabajo en equipo para que se acelerara el cambio cultural orientado a toda la empresa, para prestar un mejor servicio a los usuarios y para que el personal estuviera capacitado en la operación de equipos de alta tecnología y, con ello, alcanzar niveles internacionales que le permitieran hacer frente a las nuevas condiciones de competencia; v) el aumento de la coordinación de las distintas áreas de la empresa para orientarlas hacia un objetivo común, y vi) darle a los trabajadores la posibilidad de incrementar sus ingresos en función de su contribución a las metas de la empresa (sTRM/Telmex, 1993: 15). 
El proceso de modernización tecnológica, administrativa y organizacional tiene como uno de sus pilares la creación de áreas productivas a través de la compactación de especialidades, esto tiene como propósito la universalización, flexibilización e integración de los puestos de trabajo en función de un cambio tecnológico que haga eficiente la utilidad de la mano de obra. Como parte del interés por generar condiciones acordes con la creación de áreas productivas, se expidió un documento denominado "Modificación de los Perfiles de Puesto y de las Condiciones de Trabajo en la Organización de la Empresa”, el cual establece: i) concretar el proceso de compactación de especialidades hacia la constitución de áreas productivas; ii) concluir las negociaciones sobre reubicaciones y revisiones de perfiles en las mejores condiciones; iii) redefinir la acción sindical para la recuperación actual y nueva materia de trabajo; iv) concretar la operatividad de la Comisión de Higiene y Seguridad para el mejoramiento de las condiciones y medio ambiente de trabajo; v) orientar las ocupaciones de puestos de trabajo conforme a la necesidad de cada área, en el corto, mediano y largo plazo, y vi) establecer mecanismos para la correcta aplicación y administración de los escalafones en cada una de las especialidades y centros de trabajo (STRM, 1995: 78).

Con respecto a los cambios organizacionales de la empresa, éstos tienen que ver con el objetivo fundamental de enfocar las actividades de la organización hacia la atención y el servicio a los clientes, lo que ha implicado una profunda reorganización y cambio cultural. Además, se reestructuró la empresa en lo que se refiere a descentralización de la operación y comercialización de los servicios: se establecieron 10 nuevas Direcciones Divisionales (siete foráneas y tres metropolitanas en la ciudad de México), las que se encargan de manera integral de los procesos de comercialización de servicios, operación de la infraestructura y administración de recursos humanos y materiales.

Para desarrollar los recursos humanos de la empresa, de acuerdo con la nueva estrategia, Telmex se apoyó en los programas de capacitación impartidos por el INTTELMEX ${ }^{3}$. De los cursos imparti-

${ }^{3}$ El intTelmeX es una sociedad civil creada el 1 de septiembre de 1991 por decisión del Consejo de Administración de Telmex, a efecto de cumplir con lo dispuesto en el Convenio de Calidad, Productividad y Capacitación para la Modernización de Telmex, y dio inicio a sus actividades el 10 de noviembre del mismo año. Su creación obedeció a la necesidad de capacitación en Telmex y para cumplir con los compromisos establecidos en el título de concesión en materia de capacitación, adiestramiento, investigación y desarrollo científico y tecnológico. El INTTELMEX cuenta con una infraestructura física de 225 aulas en 13 campi, 10 salas de videoconferencias, 109 aulas auxiliares, 4 aulas móviles y 20 laboratorios de cómputo. 
dos destacan los dirigidos a la atención al cliente, sistemas de información y nueva tecnología, con el enfoque de capacitación en el lugar de trabajo (Telmex, 1991: 13).

Los resultados de cumplimiento de metas al cierre de 1994 presentaban ya los grandes alcances en la modernización en Telmex, el comportamiento global de la situación de los 1,349 centros en todo el país, se advierte en el Cuadro 1.

\section{Cuadro 1}

Cumplimiento de metas

\begin{tabular}{|c|c|}
\hline Rango de cumplimiento de meta & Total de centros \\
\hline $100 \%$ o más & 815 \\
$90 \%$ a $99 \%$ & 418 \\
Menos de $90 \%$ & 116 \\
\hline Áreas & Porcentaje global del cumplimiento \\
& de meta corporativa \\
\hline Conmutación-transmisión & $101.27 \%$ \\
Planta exterior & $99.92 \%$ \\
Tráfico & $97.98 \%$ \\
Comercial & $93.69 \%$ \\
\hline
\end{tabular}

Fuente: STRM (1995), "Informe de la comisión de modernización” en XX Convención Nacional Ordinaria Democrática.

Durante 1995 se inauguró la Red Universal de Teléfonos de México (UniNet) la cual proporciona servicios de enlace multiprotocolo nacional e internacional, con altas capacidades de enrutamiento y conmutación de paquetes que permiten funciones de conexión de servidor a servidor, terminales virtuales, correo electrónico, conversión de protocolos, videoconferencias y acceso a Internet.

Hasta la fecha, Telmex es la empresa privada mexicana con mayor número de clientes e inversionistas, en tanto que se mantiene en servicio los 365 días del año, con más de 9'200,000 líneas telefónicas terrestres o inalámbricas. Asimismo, comunica a más de 20,500 poblaciones en todo el país, maneja 7’294,000 de minutos de tráfico de larga distancia nacional y 3’024,000 de minutos de tráfico de larga distancia internacional equivalentes a 1'811,000 y 504'000,000 de conferencias, respectivamente (Telmex, 1991: 3). Lo anterior se verá afectado de alguna forma en el futuro por la apertura a la competencia, pero por las condiciones en las cuales se estableció la entrada de nuevas compañías telefónicas, y por el arraigo tradicional de Telmex en la población mexicana, al parecer seguirá siendo la principal empresa de telecomunicaciones en México, por lo menos en el futuro inmediato. 
Asimismo, en el mismo año se continuó con el crecimiento de la red local, con lo que se tuvo 8'801,030 de líneas en servicio, y se alcanzó una densidad telefónica de 9.7 líneas por cada 100 habitantes y 45.1 líneas residenciales por cada 100 hogares; se sustituyeron 720,006 líneas analógicas para lograr 87.6\% de digitalización de la planta telefónica local; se amplió la cobertura de los servicios telefónicos a 107 poblaciones adicionales, con lo que se llegó a un total de 20,554 poblaciones incorporadas a la red telefónica nacional; se impulsó el crecimiento y modernización de la planta telefónica pública al instalar 45,055 aparatos activados por tarjetas de débito de microchip; se rehabilitaron 1,524 distritos telefónicos, y se amplió la cobertura de la red telefónica celular al incorporar 93,052 clientes al servicio (Telmex, 1995: 2-5).

El propósito del gobierno era que mediante una lógica de competencia se ampliara la red telefónica sobre la base del sistema de interconexión, con lo que Telmex quedaba como la empresa central más importante en México y por lo tanto, a ella habrían de recurrir las demás empresas de telefonía que se establecieron en el país, en razón de que tendrían que rentar la red ya establecida; de este modo se mantenía el monopolio de Telmex en la prestación del servicio público de telefonía básica.

El servicio de larga distancia quedó protegido hasta 1996. A partir del 11 de agosto de ese año, se permitió la competencia en el servicio telefónico de larga distancia a las empresas que contaran con infraestructura propia y por tanto, que no requirieran de la interconexión a la red Telmex, y a partir del 1 de enero de 1997, la empresa empezó a competir con otras en la prestación del servicio, sobre la base del sistema de interconexión de redes, y quedó como la red fundamental la que está a cargo de Telmex. De ese modo, la función de ésta se modificaría al pasar de la prestación directa del servicio a rentar su red a otras compañías. ${ }^{4}$

A partir de los años ochenta y principios de los noventa del siglo xx, se empezó a discutir sobre cómo caracterizar la forma y las cualidades específicas de los cambios laborales que se dieron en Telmex en dicho periodo. Haciendo un esfuerzo de síntesis, podríamos decir que estos cambios son los siguientes: i) la apari-

\footnotetext{
${ }^{4}$ La modificación de la concesión al amparo de la cual opera Telmex se firmó con el gobierno federal el 10 de agosto de 1990, con vigencia hasta el año 2026 y con posibilidad de una renovación posterior de 15 años, establecía las normas de calidad requeridas para la prestación del servicio y definía las bases para la regulación tarifaria. La SCT fue la encargada de revisar que se cumplieran todos los acuerdos de la concesión.
} 
ción simultánea de tecnologías cualitativamente nuevas como son las basadas en la microelectrónica; ii) los nuevos requerimientos de los mercados de bienes (calidad, flexibilidad, etc.); iii) el reto cualitativamente nuevo que surge de la dimensión ecológica y que influye tanto en los productos como en la producción; iv) las nuevas estrategias de organización y de management; v) los nuevos conceptos de política de personal y de uso de la mano de obra (trabajo en grupos, círculos de calidad, control por confianza); vi) la modificación de factores determinantes para su salud financiera, tal fue el caso de la adecuación de las tarifas y del régimen fiscal, y vii) el cambio de la estructura contractual sin mutilaciones severas y con pocos reajustes de personal. Todos estos cambios indican una situación histórica decisiva que puede calificarse como revolución microelectrónica, revolución técnica o tercera revolución industrial (Pries, 1991: 62).

\section{La flexibilidad laboral en la contratación colectiva en Telmex}

Antes de analizar cómo se lleva a cabo la flexibilidad laboral en la contratación colectiva en Telmex, es importante mencionar que se están analizando los сст depositados en la Junta Federal de Conciliación y Arbitraje (JFCA) y que corresponden a los bienios 19941996, 1996-1998 y 1998-2000.5 Además de la revisión exhaustiva de dichos contratos, recurrimos también al análisis de los Reglamentos Interiores de Trabajo (RIT) y otros convenios, en tanto que algunas cláusulas de los CCT eran desarrollas con mayor precisión en dichos documentos. Asimismo, se advierte que al hablar de ССт se está haciendo referencia a la parte formal de la empresa, a través de la legitimación de las condiciones generales de trabajo en un documento de carácter legal.

De igual forma, reconocemos que seguramente existe en Telmex un sistema de usos y costumbres arraigados en los trabajadores que escapa a los clausulados de los CCT, y que muchas veces tienen mayor peso que lo estipulado en dichos contratos. Es importante reconocer que la práctica de estrategias de modernización productiva no sólo impacta los procesos de trabajo, sino también las identidades laborales, simbolismos y subjetividades, las cuales escapan a la codificación de las relaciones laborales y suceden como usos y costumbres al interior y al exterior de los pisos de trabajo. ${ }^{6}$

\footnotetext{
5 Al respecto véase STRM/Telmex, 1994; STRM/Telmex, 1996, y STRM/Telmex, 1998.

${ }^{6}$ Al respecto véase Allaire y Firsirotu, 1992; Bizberg, 1989; Courpasson, 1994; De Paula, 1993; Dubar, 1991; Dubet, 1989, Etkin y Schvarstein, 1995; Guadarrama, et al. 1998; Hualde, 1995; Margel, 1995; Trice, 1993, y Valenzuela, et al. 1992.
} 
Como ya se mencionó, fue a principios de la década de los ochenta del siglo xx cuando el paradigma productivo tayloristafordista entró en crisis e inició la transición a nuevos modos de acumulación de capital. Sin duda alguna, el rescate de la experiencia japonesa de la posguerra se convirtió en el eje articulador de las nuevas organizaciones de trabajo sustentadas en la llamada flexibilización laboral, aunque es importante mencionar que ésta no tiene un significado único. En términos generales, la mayoría de los conceptos sobre flexibilidad laboral se refieren a la reestructuración del mercado de trabajo y del proceso de trabajo, así como a la creciente versatilidad en el diseño y a la mayor adaptabilidad de la nueva tecnología en la producción (Smith, 1989: 35 ), amén de que casi en todas las definiciones se reconoce -implícita o explícitamente- un componente informal no codificado que funciona también como indicador de procesos flexibles en las relaciones laborales.

La flexibilidad en el trabajo puede estudiarse a través de dos dimensiones: la organización productiva y los recursos humanos. En la primera normalmente se utilizan como indicadores la flexibilidad numérica, ${ }^{7}$ la flexibilidad funcional ${ }^{8}$ y la participación de los trabajadores en la organización del trabajo. ${ }^{9}$ En la segunda se hace referencia a la capacitación de personal ${ }^{10}$ y a la flexibilidad salarial con relación al bienestar de los trabajadores. ${ }^{11}$

7 El término flexibilidad numérica hace referencia a la capacidad que tienen las empresas para transformar su planta de trabajadores, en función de las variaciones de la demanda externa de sus productos.

${ }^{8}$ La flexibilidad funcional se refiere al uso flexible de la mano de obra dentro de los procesos productivos, la modificación de la estructura de puestos de trabajo, la reasignación de tareas de los trabajadores en función del cambio tecnológico, la movilidad al interior de la empresa y la presencia de trabajadores polivalentes.

${ }^{9}$ Ésta se refiere a si los trabajadores están involucrados en el diseño de procesos, la división del trabajo, la forma de asignación de tareas, la capacitación, las funciones de los puestos, la estructura jerárquica, los grupos informales de trabajo, las relaciones interpersonales dentro del trabajo, la solución de conflictos y la negociación colectiva formal e informal en los centros de trabajo en cuanto a la interpretación de las normas laborales.

${ }^{10}$ La calificación en el trabajo, entendida como un concepto mediador del nivel organizacional y de las condiciones de trabajo se convierte, en el proceso de flexibilización, en un factor central que asume a la calificación como capital humano, como una exigencia del proceso de trabajo y como una instancia organizativa y de control (Carrillo, 1996: 61).

${ }^{11}$ Es importante mencionar que generalmente el bienestar de los trabajadores se ha asociado con los sistemas de remuneraciones adicionales al salario, de tal manera que una de las principales propuestas de los nuevos paradigmas productivos actúa directamente sobre éstos, a través de la llamada flexibilidad salarial. Tal indicador se refiere a la introducción de formas de retribución variable al rendimiento y a que la progresión salarial quede vinculada a este último y al aprendizaje. La flexibilidad salarial también 
Al revisar los сст de Telmex de los bienios 1994-1996, 1996-1998 y 1998-2000, se puede observar que, pese a que la flexibilidad laboral fue la principal estrategia de reestructuración productiva, ésta no implicó la puesta en marcha de políticas orientadas a la aplicación de la llamada flexibilidad numérica de personal. En los tres сст examinados es evidente que la contratación de trabajadores de nuevo ingreso es una decisión tomada bilateralmente entre empresa y sindicato, aunque es importante mencionar que la plantilla de trabajadores registrada en los ССТ analizados es constante durante la vigencia de éstos, es decir desde 1994 no ha habido contratación ni despido de trabajadores. ${ }^{12}$

La protección que hace el STRM de sus agremiados es tal que en los СCT no se ha pactado -por lo menos hasta el año 2000- cláusula alguna que haga referencia a la prevención de un periodo de prueba y de la consecuente evaluación para los trabajadores de nuevo ingreso. Primero, porque se trata de una plantilla de trabajadores inamovible, ${ }^{13}$ y segundo, porque si hubiera contrataciones el STRM cuenta con un mercado externo de trabajo ligado fuertemente a una red de informantes internos que recomiendan el ingreso de un trabajador; este último, normalmente, resulta ser un amigo, 'compadre' o familiar de quien hay sobradas muestras de capacidad y aptitud que no deben ponerse en tela de juicio, porque de hacerlo sería faltar a un código de honor establecido en los usos y costumbres en los pisos de trabajo. ${ }^{14}$

Otra de las protecciones que otorga el STRM a sus agremiados es el hecho de no permitirle a la empresa, por ningún motivo, contratar la realización de algún trabajo especial fuera de la operación normal, en tanto que ya están establecidos los tiempos y las cargas de trabajo; además la empresa está obligada a capacitar a sus trabajadores en las necesidades que surjan por la aplicación de nuevos sistemas de trabajo que requieran nuevas y más espe-

implica la negociación bilateral entre empresa y sindicato para definir o evitar despidos masivos, en la cual los trabajadores acuerdan congelar o reducir los salarios y horarios para salvar el empleo (sTPS, 1993:16).

${ }^{12}$ Pese a que generalmente las políticas de flexibilidad laboral van acompañadas del despido de un gran número de trabajadores, en Telmex no sucedió esto debido a que el STRM negoció con los representantes de la empresa su cooperación en el proceso de modernización, a cambio de que se respetara la plantilla de trabajadores existentes hasta antes de la firma de los convenios de modernización productiva.

${ }^{13}$ El número de trabajadores beneficiado por la firma del СCT del bienio 1994 1996 asciende a 47,876 y se mantiene hasta la firma del ССт del bienio 1998 - 2000.

${ }^{14}$ Pese a que el STRM y el mismo Telmex cuentan con bolsas de trabajo abiertas al público, es evidente que los mecanismos de ingreso a la compañía están sustentadas en el nepotismo de trabajadores clave allegados a las cúpulas sindicales o empresariales. 
cializadas calificaciones, de tal suerte que los trabajadores ya existentes son los únicos candidatos a llevar a cabo los trabajos especiales.

En cuanto al tiempo de trabajo, se puede observar que la jornada laboral pactada en el сст del bienio 1994 - 1996, que oscilaba en el rango de entre 45 y 48 h, descendió para los años subsecuentes al rango que va de 38 a 44 h. Lo anterior está relacionado con que, al introducir procesos de trabajo altamente informatizados, el número de horas requeridas para la realización de alguna actividad se reduce; en tanto que el trabajo pasa del trabajador a la máquina, el trabajador, por lo general, asume la supervisión o el control del proceso de trabajo. De igual forma se puede observar que el número de turnos en operación sigue en el tope de los tres turnos; en tanto que los trabajadores creen que la apertura de más turnos -con menos horas de trabajo- podría significar para ellos la porosidad de su tiempo libre provocada por la rotación de dichos turnos.

Por el lado de los días de descanso al año y a los que tienen derecho los trabajadores, se puede ver que éstos superan los siete días estipulados por la Ley Fedral del Trabajo (LFT). Asimismo, los días de vacaciones pactados en el ССт del bienio 1994 - 1996 ascendió en los ССТ 1996-1998 y 1998-2000 del rango que va de entre los seis y ocho días, al intervalo de entre los 11 y 12 días. Los datos anteriores podrían darnos evidencia para suponer que los trabajadores de Telmex han negociado la flexibilidad productiva en términos, no sólo de las nuevas cargas de trabajo, sino de los beneficios que éstas implican.

En cuanto a la flexibilidad funcional se puede decir que, todavía hace muy poco, en los ССт de muchas de las empresas mexicanas que hacían referencia a la promoción y ascenso de los trabajadores, se privilegiaba la antigüedad sobre la aptitud; no obstante que la evaluación de la capacidad debe ser individual y la empresa puede exigir los requisitos mínimos al trabajador para ocupar una vacante (Hernández et al., 2000: 77). En Telmex, pese a que la promoción a puestos superiores es decidida bilateralmente por las gerencias y el sindicato, la antigüedad es todavía uno de los factores que influye en el ascenso del personal, aunque no lo determina totalmente, en tanto que también influye la competencia, la capacitación y el periodo de prueba. Todavía no hay un acuerdo único en cuanto al papel que debe desempeñar la antigüedad en los mecanismos de promoción y ascenso a puestos superiores. Desde nuestro punto de vista se sugiere que, además de los facto- 
res mencionados, el desempeño, la participación y la puntualidad influyen de manera decisiva en la movilidad ascendente de los trabajadores.

En los Сст examinados se puede observar que existe la posibilidad de que los trabajadores sean llamados a desempeñar otros puestos o funciones distintos a los habituales, y que esta decisión es acordada por Telmex y el STRM con la finalidad de convertir trabajadores tradicionales en trabajadores polivalentes competentes en amplios campos o especialidades que engranen con las nuevas formas de producción. De igual forma, la empresa tiene la posibilidad de hacer cambios en los horarios o días establecidos de trabajo, lo anterior puede deberse a la indefinición u omisión en los СCT de Telmex en tanto que el STRM se ha mostrado indiferente a este tipo de negociaciones, por no sentir que éstas representen un retroceso en los beneficios logrados. En términos generales, se puede aseverar que la flexibilidad funcional en los ССТ de Telmex obedece a una gestión bilateral que da al STRM una considerable injerencia sobre la movilidad de los trabajadores, constatada por la medición de los siguientes indicadores: i) promoción a puestos superiores a través de mecanismos que tienen que ver con la antigüedad, la competencia, el desempeño, la capacitación, la participación, el sentido de responsabilidad, el periodo de prueba, la puntualidad y la decisión del sindicato y la empresa para dicha promoción; ii) cambios de puestos y funciones en los que existe la posibilidad de que los trabajadores sean llamados a desempeñar otros puestos o funciones distintos a los habituales, como una decisión tomada entre sindicato y empresa; iii) cambios de horario o días de trabajo consensuados entre todas las partes, y iv) cambios en sistemas de trabajo o maquinaria propuestos por la empresa, pero con autorización expresa del sindicato, así como la reubicación de trabajadores debido a tales cambios.

Otro de los indicadores que miden la organización productiva es la llamada participación del personal en la organización del trabajo y que puede medirse a través de la detección en el cuerpo del ССт, de la presencia de comisiones mixtas. En el caso de Telmex puede observarse que, pese a que no existe una comisión mixta sobre el reparto de utilidades ni de elaboración del cuadro general de antigüedad y escalafón, sí está clausulada la existencia de las siguientes comisiones: capacitación, higiene y seguridad, productividad y del Reglamentos Interiores de Trabajo (RIT).

Es interesante mencionar que en los ССт anteriores al del bienio 1994 - 1996 no existía un sistema participativo entre empresa 
y sindicato para discutir y mejorar la organización del trabajo. A partir de la aplicación de los programas de modernización productiva llevados a cabo en Telmex, se hizo evidente este tipo de sistemas, cuyo objetivo era elevar la productividad y la calidad en los productos y servicios de la empresa. Incluso, ya en los CCT examinados se detectan cláusulas vinculadas a sistemas de incentivos para premiar la participación y/o creatividad de los trabajadores en la organización del trabajo.

En cuanto a los acuerdos explícitos de modernidad productiva en Telmex se puede decir que ya sea en los ССт, los RIT o algún otro convenio se observa la existencia de acuerdos de modernización productiva entre los trabajadores y la empresa sobre cuestiones de productividad, incrementos de calidad, círculos de control de calidad, cargas de trabajo, prevención de accidentes, trabajo en equipo y desperdicio de materiales. Pese a lo anterior, es interesante mencionar que en los ССт examinados no hay evidencias que indiquen que existen funciones diferentes de las tradicionalmente asignadas al sindicato en relación con lograr cambios deseables en la organización del trabajo, ni que se prevea la creación de éstas, aunque seguramente existen acuerdos no codificados en un ССт o en un convenio de carácter legal en los que tácitamente se suponga dicha práctica.

Respecto de la dimensión de los recursos humanos es interesante mencionar que éstos no sólo son entendidos como el esfuerzo o la actividad humana, sino también comprende los conocimientos, experiencias, motivaciones, intereses personales, aptitudes, actitudes, habilidades, potencialidades y salud de la mano de obra. La modernización de los sistemas de recursos humanos no sólo beneficia a los empresarios, también da a los trabajadores la capacidad para incrementar sus competencias laborales en la medida que adquieran mejores atributos para poder competir en el mercado laboral. Así, para evaluar esta dimensión nos debemos remitir a la capacitación y al bienestar de los trabajadores, porque estos indicadores permiten a la empresa crear sistemas de producción más competitivos en los mercados nacionales e internacionales, y a los trabajadores les facilita la posibilidad de elevar su productividad, y por ende, su nivel de vida.

Se puede decir que la capacitación de los trabajadores se inscribe en la dimensión que denominamos recursos humanos en tanto que, el reconocimiento de los estímulos a la educación por parte del mercado laboral, incide positivamente en el crecimiento de la productividad y es indicativo de eficiencia en la asignación 
de recursos; de tal manera que la ausencia de estas características retarda el crecimiento de la productividad y señala un mercado laboral donde predominan otros incentivos distintos al de la acumulación del capital humano (Hernández et al., 2000: 46).

En la actualidad, hablar de capacitación en el trabajo nos lleva a pensar en los procesos de recalificación de la mano de obra supuestos en los procesos de reestructuración productiva iniciados a principios de los años ochenta del siglo xx. Es un hecho que ante las condiciones cambiantes de la producción, la mano de obra deberá adaptarse -vía la capacitación- a las nuevas formas de organización del trabajo. En términos macro, México difícilmente podrá ganar ventaja en la batalla mundial por los capitales, si basa su competitividad en una mano de obra barata, en vez de una fuerza de trabajo calificada, se trata de "tener un cambio de mano de obra a tener mente de obra" (Hope, 2001: 122).

Quizá el caso Telmex sea uno de los ejemplos más significativos de la puesta en marcha de políticas orientadas a la capacitación del personal, como un requisito sine qua non de las nuevas formas de producción flexible. En los СCT examinados se puede dar fe de que la capacitación es uno de los temas en los que han insistido empresa y sindicato, como un requisito de los procesos de modernización que dicha empresa está experimentando. Las cláusulas de estos contratos hacen énfasis en la capacitación de los trabajadores, en los cursos de adiestramiento para los trabajadores de nuevo ingreso, en los cursos de capacitación para la promoción a puestos superiores, en el otorgamiento de incentivos a los trabajadores por participar en cursos de capacitación, en que dichos cursos comprendan todas las áreas de la empresa, en que exista un sistema instituido de rotación de puestos para el aprendizaje de diferentes tareas, en la prevención de la capacitación formal de los trabajadores no en relación directa con su puesto o función, y en la existencia de un sistema integral de capacitación.

En cuanto al bienestar de los trabajadores se puede decir que una de las políticas que se evidencia en los Сст examinados es que Telmex ha tratado de reducir sus costos laborales a través de la previsión oportuna de los riesgos de trabajo, lo cual le ha dado la posibilidad de pagar sus incapacidades permanentes totales y los beneficios que reciben los familiares de los trabajadores en caso de que éstos fallezcan, en un margen superior a lo estipulado por la LFT; aunque es importante mencionar que el pago de las incapacidades temporales aún es el estipulado por dicha ley. Además del aumento en las compensaciones por riesgo de trabajo, se pue- 
de observar en los СCT analizados que los días de vacaciones y de aguinaldo otorgados al año anualmente a los trabajadores son superiores a lo estipulado por la LFT.

Sin duda Telmex, a través de algunas prestaciones como la ayuda de despensa, renta para casa, educación de hijos, caja y fondo de ahorro, préstamos, gratificaciones especiales, fondo adicional para la jubilación, facilidad para que los trabajadores puedan comprar lo que produce la empresa, transporte, deporte, seguro de vida, y ayuda por fallecimiento de algún familiar, ha tratado de incrementar los ingresos de sus trabajadores como una forma de equilibrarlos ante las variaciones cambiantes de la producción y del mercado de trabajo. Es un hecho que en los Сст de Telmex se observa un sistema de remuneraciones adicionales al salario, ligado al cumplimiento de metas que tienen que ver con la producción y/o el servicio, la calidad, la capacitación y con alguna comisión. Lo anterior nos ayuda a confirmar que en esta empresa se engloba un conjunto de ingresos monetarios recibidos por los trabajadores a través de distintos programas que tienden a la llamada flexibilidad salarial.

En cuanto a la estructura salarial que prevalece en Telmex es interesante observar cómo las categorías de puestos existentes en el tabulador de salarios guardan una proporción de ocho categorías relacionadas con 14 niveles salariales, lo cual puede evidenciar el interés de dicha empresa de poner en práctica un proceso de modernización tecnológica sustentado, entre otras cosas, por la compactación de especialidades como medida para elevar la eficiencia de su fuerza de trabajo y reducir sus costos laborales. También es interesante mencionar que las variaciones en el salario diario menor promedio en Telmex no han sido tan bruscas, en tanto que se han movido en el periodo examinado en un rango que va de uno a tres salarios mínimos. ${ }^{15}$ En cuanto al salario diario mayor promedio se observa que pasó de tres salarios mínimos a ubicarse, en los ССТ de los bienios 1996-1998 y 1998-2000, en un rango que oscila entre los seis y diez salarios mínimos. Asimismo, en el periodo analizado, la media salarial diaria promedio se ubica, en un rango de dos a cinco salarios mínimos, mientras que la moda salarial diaria alcanza, en los tres periodos estudiados, cuatro salarios mínimos.

Los resultados anteriores nos llevan a pensar que, por lo menos en Telmex, los ajustes salariales que se llevaron a cabo obede-

${ }^{15}$ Se aclara que se trata de salarios mínimos vigentes para los periodos examinados. 
cen a la puesta en marcha de una política de flexibilidad laboral que subyace a una política laboral de Estado que se da en un contexto de regulaciones y prácticas institucionales puestas en práctica hasta antes de los primeros años de la década de los ochenta del siglo xx, y cuyo objetivo fue aumentar el bienestar de los trabajadores y garantizarles una participación en las ganancias del crecimiento económico a cambio de la alianza política del movimiento sindical con el Estado (Hernández et al., 2000: 59).

\section{Corolario}

Desde un principio, el proceso de modernización en Telmex obedeció a la lógica privatizadora que ha implantado el Estado mexicano con sus empresas paraestatales. Este hecho -que responde a las características de un Estado de corte neoliberal- ha permitido la mejora y la expansión de las unidades productivas de Telmex dentro de un proceso de modernización técnico-organizativa.

A través de este dinámica, Telmex rompió con un pasado que la había calificado de obsoleta y retrasada en cuanto a innovación tecnológica y calidad en el servicio. Dicho proceso obedeció a la implantación de áreas productivas a través de la compactación de especialidades, proceso que tiene como propósito la universalización, flexibilización e integración de los puestos de trabajo de manera acorde con el cambio tecnológico, la capacitación y la especialización de la mano de obra.

En Telmex la reestructuración productiva vía la flexibilidad ha superado la autocomplacencia y se ha reconocido que no es suficiente partir del argumento que dicha empresa es rentable y está en crecimiento, sino del seguimiento de los factores que permitirán que ésta siga siendo una empresa rentable y en expansión, es decir de las condiciones del mercado, el avance tecnológico, el entorno regulatorio y las condiciones sociopolíticas.

Se garantizó, con el apoyo del STRM, el compromiso de la empresa de no despedir a ningún trabajador de la compañía, lo que evidencia que en la realidad los procesos de flexibilización laboral no necesariamente tienen que significar recortes de personal o disminuciones salariales.

En Telmex se exhortó a los trabajadores a explotar y a difundir su creatividad para que no sólo la empresa se colocara en la cima de las empresas más competitivas del mundo, sino también para que éstos disfrutaran de los beneficios derivados de la puesta en marcha de políticas orientadas a modernizar y flexibilizar el 
trabajo en la empresa. Asimismo, es un hecho que los cCT de Telmex muestran la disposición constante de la empresa para operar programas de capacitación que eleven no sólo la productividad de la fuerza de trabajo, sino también que creen conciencia acerca de la responsabilidad de ésta en los procesos de trabajo.

Otra de las cuestiones que surgen de la modernización laboral vía la flexibilidad en Telmex tiene que ver con el hecho de que esta empresa fue de las pocas que lograron integrarse a la modernidad; en tanto que a partir de los primeros años de la década de los ochenta del siglo xx, se había colocado como una de las compañías que había logrado un impacto positivo en el Producto Interno Bruto (PIB); y se había posicionado como una empresa prioritaria en la economía mexicana al incorporar tecnología de punta, ser una firma altamente innovadora y hacer partícipes a sus trabajadores de los beneficios de la nueva política de la economía neoliberal.

Es evidente que las políticas sobre flexibilización laboral puestas en práctica en Telmex no sólo significaron una mejora para los accionistas y la plantilla de trabajadores, sino que también se extendió a los 'nuevos clientes' en tanto que éstos comenzaron a beneficiarse de la expansión de los servicios en comunicación que desarrolló Telmex, sustentados en la filosofía llamada 'cliente-proveedor' y la nueva cultura corporativa que fomentaba el compromiso, la cooperación, el cumplimiento, el esfuerzo, la solución de problemas, la innovación, la competitividad, la calidad, la productividad, la desburocratización, la confianza y la lealtad.

Por último, y a manera de corolario, se puede decir que la flexibilidad laboral tiene diferentes connotaciones en tanto que depende de las estrategias empleadas por cada empresa y de las necesidades de cada una de éstas. Lo que sí es un hecho es que la noción de flexibilidad laboral debe ir más allá de una cuestión meramente de estrategia gerencial que se presenta sólo al interior de la empresa y debe ubicarse en un plano de política sindical. Cabe preguntarse: ¿hasta cuándo podrá darse la situación de mantener los puestos laborales y los incrementos salariales?, debido a que en la actualidad la innovación tecnológica y la flexibilidad laboral han permitido que el trabajo requiera de menor mano de obra. Hasta el momento, esto se ha mantenido gracias a que no se ha contratado nuevo personal y a que se ha reubicado a los trabajadores restantes en los nuevos puestos que supone la reestructuración productiva de Telmex. De no poderse mantener esta situación, el éxito de Telmex frente al proceso de modernización 
productiva y de flexibilidad laboral dejará de ser un éxito para volverse un fracaso.

\section{Bibliografía}

Allaire, Y. y M. Firsirotu (1992), “Teorías sobre la cultura organizacional”, en Cultura Organizacional: Aspectos Teóricos, Prácticos y Metodológicos, Fondo Editorial Legis, Bogotá.

Bizberg, I. (1989), “Individuo, identidad y sujeto”, en Estudios Sociológicos, vol. vil, núm. 21, El Colegio de México, México, pp. 30-52.

Carrillo, J. (1996), "Reestructuración productiva, trabajo y educación en América Latina”, en Lecturas de Educación y Trabajo, núm. 3, Organización Internacional del Trabajo, México.

Courpasson, D. (1994), “Marche concret et identité professionnelle locale: la construction de l'identité par le Rapport au Marché", en Revue Francaise de Sociologie, abril-junio, Centro National de la Recherche Scientifique, París, pp. 16-25.

De la Garza, E. (2002), La democracia de los telefonistas, Universidad Autónoma Metropolitana, México.

De Paula, M. (1993), “Innovación tecnológica y subjetividad obrera” en Sociología del Trabajo, núm. 19, Siglo xxi, España, 38-57.

Dubar, C. (1991), La Socialization: Construction des Identités Sociales et Professionnelles, Armand Colin, París.

Dubet, F. (1989), «De la sociología de la identidad a la sociología del sujeto», en Estudios Sociológicos, vol. III, núm. 21, El Colegio de México, México, pp. 5-29.

Etkin, J. y Schvarstein (1995), Identidad de las Organizaciones: Invarancia y Cambio, Paidós, Buenos Aires. 
Guadarrama, R. et al. (1998), Cultura y Trabajo en México. Estereotipos, Prácticas y Representaciones, Universidad Autónoma Metropolitana, México.

Hernández, E. et al. (2000), Productividad y mercado de trabajo en México, Plaza y Valdés Editores, México.

Hope, M. (2001), "Las chambas de la era global” en Expansión, núm. 830, México, pp. 111-120.

Hualde, A. (1995), "Ingenieros en la frontera norte de México: trayectorias laborales e identidades profesionales", ponencia presentada en el Coloquio: Tendencias y Manifestaciones de la Nueva Cultura del Trabajo, organizado por la Revista El Cotidiano, Universidad Autónoma Metropolitana-Azcapotzalco y la Fundación Friedrich Ebert, septiembre, México.

Margel, G. (1995), “Calificación laboral e identidad: un mismo rostro de la cultura de la cooperación”, en xx Memorias del Congreso Latinoamericano de Sociología, octubre, México.

Medina, I. (1995), “Teléfonos de México: modernización, privatización y nuevas relaciones laborales" en Espiral, Estudios sobre Estado y Sociedad, vol. I, mayo-agosto, Universidad de Guadalajara, México, pp. 138-147.

Pries, L. (1991), "El cambio industrial en las sociedades modernas como sociedades de riesgo", en Sociología del Trabajo, núm. 12, Barcelona, España, pp. 59-82.

Sandoval, J. (1990), “La privatización no es cosa clara en las telecomunicaciones" en Trabajo, núm. 2,Universidad Autónoma Metropolitana-Xochimilco, México, pp. 50-78.

Smith, Ch. (1989), "Especialización flexible, automatización y producción en serie", en Sociología del Trabajo, núm. 7, Nueva Época, Barcelona, pp. 35-61. 
STPS (1993), "La movilidad de la mano de obra en el sector manufacturero de México", en Cuadernos del Trabajo, núm. 3, Secretaría del Trabajo y Previsión Social, México.

STRM (1995), “Informe de la comisión de modernización”, en XX Convención Nacional Ordinaria Democrática, Sindicato de Telefonistas de la República Mexicana, México.

STRM/Telmex (1993), “Convenio de Productividad”, en Restaurador, núm. 3, Sindicato de Telefonistas de la República Mexicana/Telmex. México, pp. 11-17.

- (1994), Contrato Colectivo de Trabajo1994, Sindicato de Telefonistas de la República Mexicana/Telmex, México.

- (1996), Contrato Colectivo de Trabajo1996, Sindicato de Telefonistas de la República Mexicana/Telmex, México.

- (1998), Contrato Colectivo de Trabajo1998, Sindicato de Telefonistas de la República Mexicana/Telmex, México.

Telmex (1991), “Telmex firmó convenios con AT\&T y AlcatelIndetel” en Voces, núm. 349, iv Época, México, pp. 8-13.

(1995), Informe anual de Telmex, Telmex, México.

Trice, H. (1993), Occupational Subcultures in the Workplace, ILR Prees, Nueva York.

Valenzuela, J. M. et al. (1992), Decadencia y Auge de las Identidades: Cultura Nacional, Identidad Cultural y Modernización, Colegio de la Frontera Norte, Sonora.

Vega, G. (1994), "Modernización y cambio en las telecomunicaciones” en Voces, núm. 379, vi Época, México, pp. 6-11.

Enviado: 28 de mayo de 2002. Reenviado: 22 de noviembre de 2002. Reenviado: 28 de marzo de 2003. Aceptado: 10 de junio de 2003. 